\title{
Morphofunctional Study of the Junction Between the Left Atrium and the Pulmonary Veins in Patient with Pulmonary Hypertension
}

\author{
Jennecy Sales Cavalcanti, Laura Patrícia Ferreira Santos \\ Recife, PE - Brazil
}

Objective - To study the arrangement of the myocardial fiber bundles at the pulmonary venous left atrial junction in patients with pulmonary hypertension, and to discuss the pathophysiological importance of this element in the etiology of acute pulmonary edema.

Methods - We obtained 12 hearts and their pulmonary vein extremities from postmortem examinations of patients with the anatomicopathological diagnosis of acute pulmonary edema. The specimens, which had no grossly visible morphological cardiac alterations, were fixed in $10 \%$ formalin, and the muscular arrangement of the pulmonary venous left atrial junctions was analyzed. This material was then isolated, embedded in paraffin, underwent serial cutting (50 $\mu \mathrm{m}$ of thickness), and was stained with Azam's trichrome.

Results - We observed in our specimens that: a) the myocardial fiber bundles that originate in the atrial wall and involve the openings of the pulmonary veins were fewer than those observed in healthy material; $b$ ) the myocardial fiber bundles that extend into the pulmonary veins were shorter than those found in material originating from individuals with no pulmonary hypertension.

Conclusion - Anatomical changes that result in a reduction in the amount of myocardial fiber bundles in the pulmonary venous left atrial junction, isolated or associated with other factors, may be the cause of disorders in pulmonary circulation, leading to an increase in pulmonary venous pressure, and, consequently, to acute pulmonaryedema.

Key words: pulmonary veins, left atrium, pulmonary hypertension

Universidade Federal de Pernambuco - Recife

Mailing address: Jennecy Sales Cavalcanti - Rua Félix de Brito Melo, 912/501 51020-260 - Recife, PE, Brazil

English version by Stela Maris C. e Gandour
Pulmonary hypertension occurs when the systolic pressure in the pulmonary artery increases above $30 \mathrm{mmHg}$, hindering the maintenance of cardiac output. Of the types of pulmonary hypertension, that of stasis or reflux (venous hypertension) is frequently the most common, usually resulting from cardiac disorders that impair the normal drainage of the lesser circulation. The most common example is that of left heart failure and mitral stenosis causing pulmonary venous stasis, with a consequent increase in pressure in this region ${ }^{1,2}$. However, other causes exist, such as pathological pulmonary processes (emphysema, fibrosis, pulmonary thromboembolism, and pulmonary schistosomiasis), which explain the genesis of secondary pulmonary hypertension. However, the literature has shown that, in approximately $10 \%$ of the cases, no cardiac or pulmonary cause can be identified as being involved in the genesis of the disease; in these cases, the disorder is called primary or idiopathic pulmonary hypertension. The latter is a rare disorder of the pulmonary circulation, usually affecting young and middle-aged women, with a rapid and invariably fatal evolution ${ }^{3}$. Stuart ${ }^{4}$ reported that the veno-occlusive pulmonary disease occurs in less than $10 \%$ of patients with primary pulmonary hypertension, and that the basic cause of this disorder remains unknown.

On the other hand, several authors have reported that the myocardial fiber bundles around the openings and in the walls of the pulmonary veins in different animals, man included, have a function similar to that of a sphincter, whose action would prevent blood from reflowing from the atrium to the lungs during atrial systole ${ }^{5-9}$. Some authors have formulated the hypothesis that the contraction of the pulmonary veins associated with the cardiac activity would facilitate left atrial filling during diastole ${ }^{6,10,11}$. Other authors have reported that the myocardial fiber bundles in the walls of the pulmonary veins have a function in the regulation of the pulmonary venous pressure and of the blood flow through alterations in their tonus caused by various physiological or pharmacological stimuli, or both ${ }^{5,6,12,13}$. These authors have suggested the possibility that the constriction of the pulmonary veins or morphological alterations in 
the pulmonary venous left atrial junctions may be the cause of the acute pulmonary edema with no cardiac ventricular failure.

Considering the hypotheses formulated by these authors, in which alterations in the intrapulmonary venous pressure might be caused by modifications in the structural elements that constitute pulmonary venous left atrial junctions, we decided to use stratigraphy to investigate pulmonary morphology and function during abnormal conditions, and discuss the possible pathophysiological importance of our findings in the etiology of acute pulmonary edema.

\section{Methods}

We studied 12 anatomical specimens constituted of hearts and lungs obtained from autopsies performed at the Department of Pathology of the CCS in the Federal University of Pernambuco. These autopsies were carried out in adult female and male patients, whose anatomicopathological diagnosis of the cause of death was acute pulmonary edema. It is important to emphasize that only specimens with no grossly visible alterations were included in this study.

The hearts were withdrawn with their pulmonary vessels and part of their pulmonary parenchyma, and were fixed in 10\% formalin. Then, the left atria with a segment of the pulmonary veins were separated from the corresponding ventricles. With the aid of a stereoscopic magnifying glass, the remaining pieces of the pericardium were removed, and the myocardial fiber bundles were exposed. The pulmonary venous left atrial junction and the extension of the cardiac muscle into the pulmonary veins were carefully analyzed.

Once the pulmonary veins and their junctions with the atrial wall were isolated, the material was embedded in paraffin, and tangential and longitudinal serial cross-sectional samples of the entire specimen $50 \mu \mathrm{m}$ thick were cut. The slides were stained with Azam's trichrome, and were examined under epi- and transillumination, using a microscope and a stereomicroscopic magnifying glass with magnifications ranging from 5 to 50 times. The results obtained were recorded and illustrated by means of sketches and photographs.

\section{Results}

The myocardial fiber bundles were found to originate in the atrial wall and encompass the openings of the pulmonary veins, constituting a type of sphincter (fig. 1). Part of these fiber bundles continues in the walls of the veins in semicircular and oblique trajectories, in a spiral manner (fig. 2). This behavior was better evidenced in the superior pulmonary venous left atrial junctions than in the inferior ones, in which most fibers are limited to encircling the openings of the pulmonary veins. In both cases, we observed that some of the outermost bundles encompass the veins close to their openings, and return to the atrial wall, constituting true muscle loops. However, most fiber bundles end in the venous wall, where they attach to the fibrous elements of the vessel's

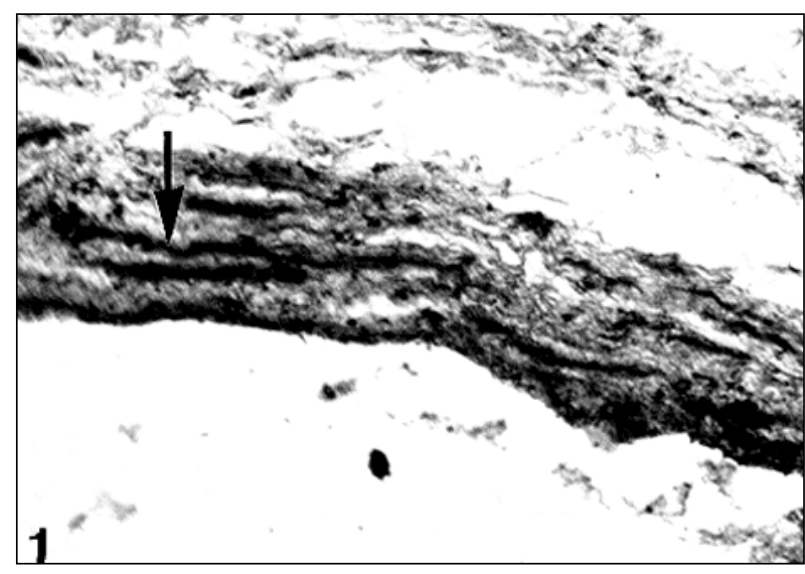

Fig. 1 - Cross-section at the level of the opening of the pulmonary vein in the left atrium. Note the bundles of myocardial fibers with semicircular trajectories around the opening of the left pulmonary vein (arrow). Azam's trichrome, 45x.

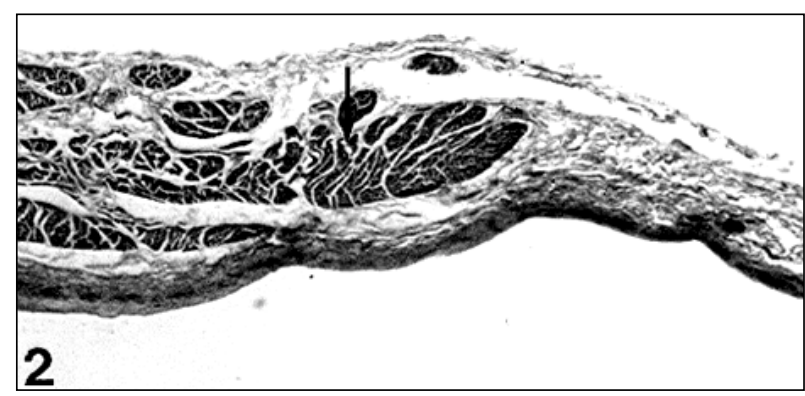

Fig. 2 - Longitudinal section at the level of the pulmonary venous left atrial junction. Note the bundles of myocardial fibers with oblique trajectories, originating from the left atrium and extending to the wall of the pulmonary vein (arrow). Azam's trichrome, 45x.

adventitia. These myocardial cuffs are limited to the tunica adventitia, and extend on an average of $10 \mathrm{~mm}$ into the superior pulmonary veins and $3 \mathrm{~mm}$ into the inferior ones, being restricted to the intrapericardial part of the vessels.

\section{Discussion}

Several authors have proposed that the cardiac musculature encompassing the openings of the pulmonary veins has a sphincteral function, which prevents blood from reflowing during atrial systole ${ }^{5-8,14-16}$. On the other hand, systolic contractions of the extrapulmonary portions of the pulmonary veins have been shown in the rat and in the mouse ${ }^{11,17}$. Likewise, Carrow and Calhoun ${ }^{10}$, in their experimental studies, formulated the hypothesis that the conduction of the electrical impulse following a trajectory established by the cardiac muscle in the wall of the pulmonary veins may create a peristaltic or milking action towards the heart, therefore, increasing the venous return and atrial filling. Nathan and Eliakim ${ }^{6}$ reported that the presence of striated muscle in the tunica media of the pulmonary veins of the rat has led to the supposition that its contraction associated with the activity of the cardiac muscle facilitates atrial filling during diastole. Likewise, Almeida et al ${ }^{17}$ suggest that the mechanical weakening or failure of the myocardial layer in 
the walls of the pulmonary veins could have an increased effect on the pressure of the pulmonary capillaries, favoring stasis and pulmonary edema. Cavalcanti et al ${ }^{18}$ reported that the pulmonary venous left atrial junction has a morphological substrate capable of playing an important role in the pulmonary circulation, not only preventing venous reflow, but also controlling intrapulmonary venous pressure and cardiac performance.

The semicircular myocardial fiber bundles that encompass the openings of the pulmonary veins observed in this study are fewer than those reported by Cavalcanti et al ${ }^{18}$ in their study analyzing the material from individuals with no pulmonary hypertension. Likewise, the extension of the myocardial cuff of the tunica adventitia of these vessels is, on average, shorter than that reported by Nathan and Elia$\operatorname{kim}^{6}$ and by Cavalcanti et al ${ }^{8}$ in non- pathological conditions. This is because the myocardial fiber bundles had not reached the pericardial reflection in any of our cases, leading us to assume that the reduction in the amount of myocardial fiber bundles encompassing the opening of the pulmonary veins may hinder their possible sphincteric function reported by several authors ${ }^{6-9,15-18}$. This might be the cause of blood reflow during atrial systole.
On the other hand, we could assume that a shorter myocardial cuff in the wall of the pulmonary veins would weaken the probable milking function of these fiber bundles, as already reported by several authors ${ }^{7,11,14}$. This would elevate pressure, causing blood stasis in this vasculararea.

It is noteworthy that an important limiting factor of the present study is the nonexistence of clinical data confirming the presence of pulmonary hypertension during life in the patients studied. In addition, our results were compared in a subjective manner with those of the control group, which was constituted of material originating from autopsies of adult female and male patients with different causes of death, those of cardiopulmonary origin excluded ${ }^{18}$.

In conclusion, anatomical alterations resulting in a reduction in the amount of myocardial fiber bundles in the pulmonary venous left atrial junction, isolated or in association with other factors, may be the cause of disturbance in the pulmonary circulation, resulting in an increase in pulmonary venous pressure, and, consequently, in acute pulmonary edema.

\section{References}

1. Bevilacqua F, Benssonssan E, Silva JMJ, Castro FS, Carvalhaes LP. Manual de Fisiopatologia Clínica. Rio de Janeiro: Atheneu, 1975: 277.

2. Chazova I, Robbins I, Loyd J, et al. Venous and arterial changes in pulmonary veno-occlusive disease, mitral stenosis and fibrosing mediastinitis. Eur Respir J 2000; 15: 116-22.

3. Veeraraghavan S, Koss MN, Sharma OP. Pulmonary veno-occlusive disease. Curr Opin Pulm Med 1999; 5: 310-3.

4. Stuart R. Hipertensão pulmonar primária. In: Harrison TR edt. Medicina clínica $12^{\mathrm{a}}$ ed. Rio de Janeiro: Guanabara Koogan, 1992: 60-3.

5. Burch GE, Romney RB. Functional anatomy and throttle valve action of the pulmonary vein. Am Heart J 1954; 47: 58-66.

6. Nathan H, Eliakim M. The junction between the left atrium and the pulmonary veins: a antomic study of human hearts. Circulation 1966; 34: 412-26.

7. Kay JM. Pulmonary vasculature and nerves: comparative morphologic features of the pulmonary vasculature in mammals. Am Rev Respir Dis 1983; 128: 353-7.

8. Cavalcanti JS, Oliveira ML, Biazotto W, Camargo AM. Morphofunctional study of the left atio-venous junctions in man. Braz J Morphol Sci 1996; 13: 25-30.

9. Hashizume H, Tango M, Ushiki T. Three-dimensional cytoarchitecture of rat pulmonary venous walls: a light and scanning electron microscopic study. Anat Embryol (Berl) 1998; 198:6: 473-80.
10. Carrow R, Calhoun ML. The extent of cardiac muscle in the great veins of the dog. Anat Rec 1964; 150: 249-56.

11. Hooker CW, McAlister HA, Ellis FW. Active contraction of the large thoracic veins in certain mammals. Anat Rec 1964; 148: 2927.

12. Smith JD, Coxe JW. Reactions of isolated pulmonary blood vessels to anoxia, epinephine, acetylcholine and histamine. Am J Phisiol 1951; 167: 732-7.

13. Eliakim M, Aviado DM. Effects of nerve stimulation and drugs on the extrapulmonary portion of the pulmonary vein. J Pharmacol Exp Therap 1961; 133: 304-12.

14. Rudolph AM, Gootman NL, Golinko, RJ. Observations on a sphincter mechanism at pulmonary venous left atrial junction. Circulation 1961; 24: 1027.

15. Hyman AL. The pulmonary veins. Ann Rev Med 1966; 17: 431-66.

16. Kjelberg SR, Olsson SE. Roentgenologic studies of the sphincter mechanism of the caval and pulmonary veins. Acta Radiol Stockh 1954; 41: 487-96.

17. Almeida OP, Böhm GM, Carvalho MP, Carvalho AP. The cardiac muscle in the pulmonary vein of the rat: a morphological and electrophysiological study. J Morph 1975; 4: 409-34.

18. Cavalcanti JS, Biazotto W, Tavares DS, et al. Anatomia funcional da junção entre átrio esquerdo e veias pulmonares. Arq Bras Cardiol 1994; 62: 239-41. 